\title{
1. Methodological Considerations on Chance Discovery
}

\author{
Helmut Prendinger and Mitsuru Ishizuka \\ Department of Information and Communication Engineering \\ Graduate School of Information Science and Technology \\ The University of Tokyo \\ 7-3-1, Hongo, Bunkyo-ku, Tokyo 113-8656, Japan \\ email: \{helmut,ishizuka\}@miv.t.u-tokyo.ac.jp
}

This paper investigates the methodological foundations of a new research field called chance discovery, which aims to detect future opportunities and risks. By drawing on concepts from cybernetics and system theory, it is argued that chance discovery best applies to open systems that are equipped with regulatory and anticipatory mechanisms. Non-determinism, freedom (entropy) and open systems property are motivated as basic assumptions underlying chance discovery. The prediction-explanation asymmetry and evaluation of chance discovery models are discussed a fundamental problems of this field.

\subsection{Introduction}

Several researchers within the Knowledge Discovery in Databases (KDD) community (e.g., Ohsawa [1.9]) questioned whether the methods of this research field are able to find what they call 'chances'. Chances refer to phenomena that might have a (high) impact to the scientific (and human) society or an enterprise in the future. High impact is intended to have two complementary readings: on the one hand it refers to opportunities, i.e., the possibility to bring about desirable effects; on the other it refers to risks, i.e., possible threats to an enterprise or society. The notion of chance discovery has been coined to cover both aspects. Finding future features is seen in contrast to prediction (e.g., in KDD), the scientific activity to derive phenomena that appear at some future time point. By contrast, chance discovery explicitly integrates human initiative into the discovery process.

Procedurally, chance discovery can be seen as a two-step activity. The first step involves a actual discovery of a certain phenomenon. The second step suggests actions taken as a consequence of a designated phenomenon (chance), which is often called (chance) management and involves supportive measures in the case of opportunities as well as preventive measures in the case of risks.

Although there might be some interesting interactions with the probabilistic notion of chance, this reading is not intended in chance discovery. Likewise, chance discovery is not concerned with discovery by chance, such as the discovery and isolation of penicillin by Alexander Fleming. 
We will discuss the following topics. In the following section, the notion of open system is explicated in terms of cybernetics and system theory, and the possibility of prediction is discussed for both nature and open systems. The next section discusses chance discovery in open systems. In particular, the notion of 'anticipation' is introduced as a mechanism for chance discovery and exemplified by examples. After that, we explicate notions underlying the possibility of chance discovery: uncertainty and freedom. In the following section, chance discovery is contrasted with KDD. Finally, we briefly discuss and conclude the paper.

\subsection{Nature vs. Open Systems}

To clarify the application field of chance discovery, we draw a broad distinction about the object of investigation: nature vs. open systems [1.12]. Whereas nature is governed by natural laws, open systems are typically modeled abstractly by cybernetics [1.1] and system theory [1.16]. Examples of open systems include 'living' systems such as human beings, scientific communities and companies, and artificial (or technical) systems, e.g., cars and power plants. Both kinds can be described by the following system-theoretical $(S 1-2)$ and cybernetical $(C 1-2)$ features (Schurz [1.12]):

$S 1$ Open systems are physical ensembles placed into an environment significantly larger than themselves. There is a continuous exchange of energy between system and environment. The environment may satisfy the system's 'needs' (see $C 1$ ) or 'destroy' the system (see $C 2$ ).

$S 2$ Open systems preserve a relative identity through time, called their dissipative state.

$C 1$ The identity in time is abstractly governed by ideal states (or norm states) which the system tries to approximate, given its actual state.

C2 Regulatory mechanisms compensate disturbing influences of the environment, i.e., they continuously try to counteract influences that move the system apart from its ideal state. If the external influences exceed a 'manageable' range, the system is destroyed.

For our present discussion, the regulatory mechanisms of open systems are of central concern since they can actively interfere with the evolution of the system, by bringing about (an approximation of) the ideal state, or avoid the destruction of the system. Later, we will introduce a new kind of mechanism, called 'anticipation', that has the potential to significantly influence the systems evolution and most closely corresponds to our notion of chance discovery.

\subsubsection{Prediction in the Natural Sciences}

Nature is governed by the laws of physics, e.g., Newton's second axiom (the total force law). Obviously, in the physics domain there is no way to influence 
the natural laws. So even if we predict a phenomenon of high impact to society, such as a giant meteorite approaching the earth at high speed, all we can do is to evacuate the area the meteorite is predicted to hit.

Since it is not possible to change the course of nature, chance discovery here means to take appropriate (supportive, preventive) measures to minimize damage or maximize benefit.

\subsubsection{Prediction in Open Systems}

Open systems are characterized by system laws. Schurz [1.12] argued that we are theoretically unable to determine the exact numerical values corresponding to system laws, because the systems are open and hence described by nonlinear differential equations. In the extreme case, if external influences exceed the manageable (or critical) range of the system, nonlinear dynamics becomes effective and leads to chaotic behavior. Due to the sensitivity of open systems to external influences, prediction is a difficult matter. Below we will argue that in open systems, the activity of regulatory mechanisms is of major importance, rather than prediction.

\subsection{Chance Discovery in Open Systems}

\subsubsection{Enterprise Example}

Let us first give an illustrative example. Enterprises (companies) can be viewed as open systems that consist of subsystems (branches, sections, and individuals), and operate in an environment, the so-called 'economic market'. This environment typically satisfies the companies 'needs', e.g., customers demand the company's products. Under unfortunate circumstances, the company may run into the risk of being 'destroyed', e.g., by the appearance of a strong competitor (cf. $S 1$ ). In spite of that, companies preserve identity through time (cf. $S 2$ ). A company constantly tries to approximate an ideal state where, for instance, increasing profits are made and the economic situation of the company is stable. This is achieved by the company's subsystems that perform certain functions, including good production and distribution, and marketing (cf. C1). A company is typically confronted with a multitude of 'disturbing' influences in the form of, e.g., cheaper and better products of other companies and changing customer needs. At this point, the regulatory mechanisms of the company come into force, e.g., to lower production costs by increasing the efficiency of the production cycle. It is well-known that companies go bankrupt when a critical range is exceeded (cf. $C 2$ ).

\subsubsection{The Limits of Regulatory Mechanisms}

Regulatory mechanisms are the system's means to approximate the system's ideal state. Those mechanisms are mainly active to compensate disturbing 
influences by reacting to them. Although regulatory mechanisms are usually able to guarantee the identity of an open system, they come into force only if confronted with 'threats' from the environment. For instance, if a company's sales decrease, the CEO might decide to shrink the company, thereby making a number of people unemployed.

In the next section we will argue that in addition to regulatory mechanisms, open systems need mechanisms of anticipation to cope with the complexities and influences of the environment.

\subsubsection{Chance Discovery as Anticipation}

In a recent report to the Club of Rome, Botkin et al. [1.2] introduce the term "anticipation" as a key feature of innovative learning that emphasizes human initiative. It is described as follows [1.2, p. 25]:

[...] anticipation is not limited to simply encouraging desirable trends and averting potentially catastrophic ones: it is also the "inventing"

or creating of new alternatives where none existed before.

Anticipation is contrasted to prediction, since the former focuses on the creation of possible and desirable futures, and plans to bring them about. The notion of anticipation shares the intuition of Alan Kay's phrase "The best way to predict the future is to invent the future".

Promotion In philosophy of science, the term "self-fulfilling prophecy" describes situations such as the following. Newspapers write articles about the morbidity of a bank institute. As a consequence, many customers of this institute withdraw their money and other commitments. In effect, the bank institute gets into serious trouble. A recent 'real' example is the success of the so-called New Economy (internet and telecommunication related shares). Since many people believed in its success, it became a great success (at least for some time).

Chance discovery as anticipation in this context means the promotion of a trend desired by New Economy companies. As a result of promotion, the desired trend could be effected. Similar forms of promotion are daily practice in companies: certain products are advertised with the hope that they actually trigger a desire in customers. The detection of 'latent' customer desires will be briefly discussed in the next section.

Collaboration In business there is a lot of talk about 'mergers'. Collaborations are also seen in scientific research programs. We will briefly describe the field of Quantum Computation.

Deutsch [1.3] is reported to be the first to explicitly ask whether it is possible to compute more efficiently on a quantum computer. For a long time, this possible collaboration of quantum theory (physics) and artificial intelligence (computer science) remained a curiosity. However, there are already some indications of 'killer applications' for quantum theory. For instance, Spector 
et al. [1.13] report on problems that take polynomial time on a quantum computer but exponential time on a classical computer.

In academics, possibilities for collaborations are ubiquitous, and sometimes realized, e.g., in genome analysis, artificial intelligence and biology collaborate. What might chance discovery as anticipation mean here? In particular, how can we anticipate the success of a certain kind of collaboration? We cannot provide a working methodology here. In the case of quantum computation, the chance was 'discovered' by Feynman [1.5] who observed that classical systems cannot effectively model quantum mechanical systems. This observation suggests that computers based on the laws of quantum mechanics (instead of classical physics) could be used to efficiently model quantum mechanical systems, and possibly even solve classical problems such as database search in a highly efficient way.

Given that Quantum Computation will indeed be successful, how could we have known 10 years ago? One method would be to track the history of 'conjectures' (ideas, observations) formulated by various insightful researchers, and evaluate their feasibility in the light of current knowledge in possibly quite different research areas. The availability of huge amounts of information on the Web might facilitate such an endeavor.

\subsection{Chance Discovery, Uncertainty, Freedom}

One of the tacit assumptions underlying chance discovery is that the future is uncertain, and hence there is freedom to change is course of action. For the sake of argument, assume the opposite, i.e., the world history evolves deterministically. Obviously, under this artificial assumption, chance discovery (in our sense) is not possible as there are no choices.

\subsubsection{Freedom}

Following [1.15], we propose entropy as the measurement of freedom. Specifically, the measurement of freedom is phenomenologically rather than procedurally oriented. The freedom of a set $A$ of alternatives is measured by the entropy $H$ of the actual chosen proportions, i.e.,

$$
H(A)=-\sum_{i \in A} p_{i} \log p_{i}
$$

where $\log$ is to the base $2, p_{i} \geq 0$ and if $p_{i}=0$ then $0 \log 0=0$. Accordingly, we may say that chances exist if there are (almost) evenly distributed alternatives. Consider the following situations (A) and (B).

(A) There are three sellers with (approximately) 30\% market share.

(B) There are two sellers, one has $75 \%$, the other has $25 \%$ market share. 
Situation (A) has more freedom than situation (B), since a market with one dominant provider has low entropy. The more interesting notion here is freedom of successive states for a number of time periods. For instance, a market with $100 \%$ customer loyalty is not free.

\subsubsection{Explaining versus Predicting}

Let us recall the aforementioned open system situation, that features a high degree of uncertainty, and formulate it as a problem for chance discovery and chance management (CD\&CM). In the following, $M$ stands for a CD\&CM model (or theory).

- Assume as given a model $M$ that explains why a particular phenomenon $X$ turned out to be a chance (opportunity or risk), as observed by its high (positive or negative) impact.

- Given a phenomenon of type $X$, can we employ $M$ to predict high impact under comparable circumstances?

Of course, the notions of phenomenon of type and comparable warrant further explication. In order to clarify the problem, consider the case of simple unstable or chaotic systems that support explanations without predictive value. Assume an ideal ball exactly on top of another ideal ball. Here, we cannot predict in which direction the ball will roll down, but after it rolled down, we can explain it by an unmeasureably small disturbance in the direction in which the ball rolled down [1.11].

Thus, the 'explanation vs. prediction' problem raises the fundamental question about which systems support the predictive use of chance discovery results. Straightforward answers seem to be ruled out by the fact that human initiative is essential to take opportunities or avoid risks, and the complexity of systems such as the web or financial markets.

As a more realistic example, consider Ogawa's [1.7] ILE (Information of Liability and Equity) measure that identifies risk factors that eventually lead to bankruptcy. Specifically, ILE explains bankruptcy. The crucial question, however, as in science is whether ILE can predict bankruptcy. If ILE has predictive value, the impact of preventive measures can be proven. Given the theoretical result about the infeasibility of prediction in open system, we are left with a probabilistic notion of prediction.

\subsection{Scientific Evaluation of Theories}

A basic question about scientific theories is how they can be evaluated. Following Popper [1.10], a theory is corraborated (or validated) if it predicts a phenomenon that is actually observed, while it is falsified when a phenomenon is observed that contradicts the observation. Note that a theory can never be 
verified by a finite set of observations. The situation for CD\&CD models is complicated for the following reason.

Triple-theory Problem Whether the discovery of a potential chance turns into a positive result is dependent on three factors:

1. The designated phenomenon was a 'real' chance, i.e., chance discovery is successful.

2. The chosen measures were appropriate, i.e., chance management was successful.

3. The predictions about the world for the associated time span of CD\&CD were sufficiently accurate.

The triple-theory problem refers to the practical problem that in order to validate (or falsify) a CD\&CM model, three sub-theories have to be successful. If all of them are successful, observed by the positive result, the model is corraborated. However, in the case of a negative result, we cannot simply say that the designated phenomenon was no chance, because we either did not choose appropriate (supportive or preventive) measures to bring about the positive outcome or our predictions about the boundary conditions for the positive outcome have been false.

From a methodological point of view, the triple-theory problem puts serious doubts whether we might be able to evaluate CD\&CM models scientifically. Due to the very nature of the open systems, reproducibility of results is infeasible.

\subsection{Chance Discovery vs. KDD}

Fayyad et al. [1.4] characterize Knowledge Discovery in Databases (KDD) as

[...] the nontrivial process of identifying valid, novel, potentially useful, and ultimately understandable patterns in data.

The discovery goal in KDD can be divided into a descriptive and a predictive part. In description the system seeks for patterns (or models) in order to present them to the user in an intelligible way; in prediction the system finds patterns so that the future behavior of some entity can be predicted. There exist a number of established (mostly statistical) data mining methods to achieve those goals, such as classification, regression, clustering, summarization, dependency modeling, and change and deviation detection [1.4].

Chance discovery may use the knowledge extracted by data mining methods to detect future features. For instance, by Web usage mining, i.e., the clustering of Web users based on their browsing activities, potential customer groups can be identified, and specifically addressed by companies. Here the interplay of data mining - describing correlations between users' interests - and chance discovery - actively promoting a possibility —is of crucial importance. 
One may ask whether, e.g., data mining already is a form of chance discovery. Our answer is "no". Data mining can summarize or predict trends, but leaves out the rôle of human interference. Anticipation as a mechanism of an open system, on the other hand, 'matches' a desired (or predicted) trend with the system's goals (typically human 'desires') and accordingly takes supportive or preventive measures.

Another way of contrasting Chance Discovery and KDD is as follows. Whereas KDD tries to detect most likely trends in data, Chance Discovery aims at finding data that do not match likely patterns but indicate interesting phenomena not yet exploited and bearing potential of future trends. However, currently there exist no serious analysis to distinguish those high-potential phenomena from 'noise' in data. Basically, this means that exceptions can be equally informative as highly probable regularities. As an example, consider the following. Humans that are infected with plasmodium vivax are very likely to contract malaria. However, some people do not. In KDD terms, those people are ignored since they do fall under the likely case (contracting malaria). It turned out that it is due to a special genetic constellation that some people have a strong protection against malaria. In Chance Discovery terms, the explanation of those people's resistance against malaria is a chance for a significant scientific discovery.

\subsection{Discussion and Conclusion}

In this paper, we explicate our take on a new research area called 'Chance Discovery'. The notion of 'open system', as characterized in cybernetics and system theory, serves as a framework to embed the activity of Chance Discovery. In particular, anticipation is introduced as a mechanism that may perform the rôle of detecting chances in open systems. The anticipating mechanism is explained in the context of promotion in New Economy and collaboration in the Quantum Computation research programme. Chance Discovery is contrasted to KDD and mutually beneficial aspects are explained. We identify human initiative as a distinguishing feature of Chance Discovery (as opposed to KDD), e.g., to actively initiate and foster a trend by promotion or to actively explore the (practical) feasibility of a theoretical conjecture.

Unlike the practical methods for data mining, we only described a methodology for Chance Discovery. A method for Chance Discovery might analyze 'success stories', i.e., cases where features of high impact for the future were successfully identified and accordingly promoted by human initiative. This retrospective analysis might be framed and processed by means of KeyGraph [1.8], a smart indexing method originally developed for information retrieval.

Recently, McBurney and Parsons [1.6] proposed principled methods to discover chances based on dialogue games. In the context of e-commerce systems, Stolze and Ströbel [1.14] investigate interviews with buyers in order 
to identify their (implicit) needs. We believe that the theoretically founded methods will have the greatest impact on the field of Chance Discovery.

In this paper, we mainly focussed on the epistemological aspect of chance discovery. However, the discovery of potential opportunities and risks seems to be intimately connected to questions about human values, what should be the case and what should not be the case. Obviously, there are no opportunities or risks per se, they are only given with respect to certain values and associated goals of humans. To give drastic example, the detection of a future earthquake is not only a high risk for people living in a particular region, it is also an opportunity for certain organizations to take advantage of the chaos following the earthquake.

\section{Acknowledgments}

We would like to thank the reviewers for their sincere and valuable comments and suggestions. This research is supported by a JSPS Research Grant (19992003) for the Future Program.

\section{References}

1.1 Ashby, W. R. (1964) An Introduction to Cybernetics. London.

1.2 Botkin, J. W.; Elmandjra, M.; and Malitza, M. (1998) No Limits To Learning. Bridging The Human Gap. A Report to the Club of Rome. Pergamon Press.

1.3 Deutsch, D. (1985): Quantum theory, the Church-Turing principle and the universal quantum computer. In Proceedings of the Royal Society of London, 97117.

1.4 Fayyad, U.; Piatetsky-Shapiro, G.; and Smyth, P. (1996): Knowledge discovery and data mining: Towards a unifying framework. In Proceedings 2nd International Conference on Knowledge Discovery and Data Mining (KDD-96).

1.5 Feynman, R. (1982) Simulating physics with computers. International Journal of Theoretical Physics 21:467-488.

1.6 McBurney, P.; Parsons, S. (2001): Chance discovery using dialectical argumentation. In Y. Ohsawa (ed.), Proceedings of the First International Workshop on Chance Discovery, 37-45.

1.7 Ogawa, S. (2000): Building of trust evaluation model based on the failure prediction. In Y. Ohsawa (ed.), Workshop on Chance Discovery and Management. In conjunction with KES'2000, Brighton, UK.

1.8 Ohsawa, Y.; Benson, N. E.; and Yachida, M. (1998): KeyGraph: automatic indexing by co-occurrence graph based on building construction metaphor. In Proceedings Advanced Digital Library Conference (IEEE ADL-98), 12-18.

1.9 Ohsawa, Y., ed. (2000) Workshop on Chance Discovery and Management. In conjunction with the Forth International Conference on Knowledge-based Intelligent Engineering Systems and Allied Technologies (KES'2000). Brighton, UK: IEEE, Inc.

1.10 Popper, K. (1963) Conjectures and Refutations. Routledge and Keagan Paul. London. 
1.11 Schurz, G. (1995): Scientific explanation: A critical survey. Foundations of Science 3:429-465.

1.12 Schurz, G. (1999): Normic laws as system laws: Foundations of nonmonotonic reasoning. In Proceedings 4th Dutch-German Workshop on Nonmonotonic Reasoning Techniques and Their Applications (DGNMR-99).

1.13 Spector, L.; Barnum, H.; Bernstein, H. J.; and Swamy, N. (1999): Quantum computation and AI. In Proceedings 16th National Conference on Artificial Intelligence (AAAI-99). Invited Talk.

1.14 Stolze, M; Ströbel, M. (2001): Utility-based decision tree optimization: A framework for adaptive interviewing. In Proceedings 8th International Conference on User Modeling (UM-01), 105-116.

1.15 Suppes, P. (2000) Freedom and uncertainty. In Natke, H., and Ben-Haim, Y., eds., Uncertainty: Models and Measures, Mathematical Reasearch. Academie Verlag. 69-83.

1.16 v. Bertalanffy, L. (1979) General System Theory. New York. 